\title{
Where are we now in diabetic research?
}

\author{
Mi-Kyoung Kwak $\cdot$ Hunjoo Ha
}

Published online: 2 February 2013

(c) The Pharmaceutical Society of Korea 2013

Diabetes along with obesity has emerged as a silent epidemic worldwide owing to sedentary lifestyle and high calorie diets. This is certainly the case in Korea, where diabetes and pre-diabetes (impaired fasting glucose) accounted for 10.1 and $19.9 \%$ of the population aged over 30 years or older, respectively, in 2010 (Diabetes Fact Sheet in Korea 2012). Likewise, in the United States, diabetes accounted for 25.8 million children and adults, $8.3 \%$ of the population in 2010 (National Diabetes Fact Sheet 2011, USA). The physical and economic burden of diabetes to both the patient and society are enormous. Current anti-diabetic drugs with different mode of action have limited effectiveness and tolerability, and diabetic patients under current treatment modality will progress to micro- and macro-vascular complications. The prevalence of diabetes is predicted to rise continuously, and more people will experience diabetic complications unless new and more effective therapeutic and preventive measures become available.

A large body of information has accumulated and important progress has been made in the last decade in our understanding of molecular mechanisms involved in the development and progression of insulin resistance, insulin secretion ( $\beta$-cell function), and diabetic complications. These include autophagy, endoplasmic reticulum (ER)

\section{M.-K. Kwak}

College of Pharmacy, The Catholic University of Korea,

Gyeonggi-do, Bucheon, South Korea

e-mail: mkwak@catholic.ac.kr

\section{H. Ha $(\square)$}

Department of Bioinspired Science, Division of Life and Pharmaceutical Sciences, College of Pharmacy, Ewha Womans University, Seoul, South Korea

e-mail: hha@ewha.ac.kr stress, metabolic sensors, abnormalities in metabolites formation and transcriptional machinery associated with glucose/lipid toxicity, dysregulation of adipokines, and chronic low grade inflammation. In addition, the discovery of microRNAs (miRNAs), short single-stranded RNA molecules comprising about $1 \%$ of the genome and regulating target mRNA translational repression or cleavage, has increased our understanding on the pathogenesis of diabetes and its complications. Considering that diabetes has a strong genetic predisposition, the availability of genome-wide association (GWA) and exome sequencing studies will certainly improve our insights on genetics of diabetes and its complications.

It is true, however, that effective treatment of diabetes and prevention of diabetic complications remain elusive goals. For future direction of research in diabetes to achieve a more effective correction of intracellular metabolic errors through exploration of new therapeutic targets, it is appropriate that we review current status of research in diabetic research.

This special issue starts from the review by Cheon (2013), which provides the latest information about the novel molecular targets of type 2 diabetes mellitus (T2DM) pathogenesis and current development status of new T2DM therapeutics (Cheon 2013). Particularly, it highlights new therapeutic approaches, including incretin-based therapy, renal tubular glucose transporter inhibitors, $\beta$-hydroxysteroid dehydrogenase 1 inhibitors, peroxisome proliferator-activated receptor modulators, regulators of glucose/ lipid metabolism, and anti-inflammatory therapies. The next series of reviews deal with novel pathophysiological mechanisms of T2DM as an intension to seek for the novel therapeutic strategy. The second article by Lee (2013) and colleagues provides reviews on recent findings related to the roles of miRNAs in T2DM (Park et al. 2013a). The 
miRNAs are directly involved in the post-transcriptional regulation of specific genes and have thereby emerged as an important regulator of diverse biological processes, including pathological changes. In the earlier part, the review provides basic concepts of miRNAs, including their biogenesis and general mechanism of action, and then introduces in-depth information of miRNAs, which are associated with pancreatic $\beta$-cell development/function, hepatic metabolism, muscle insulin sensitivity, and lipid metabolism/adipogenesis. This rapidly growing miRNA area sheds light on the potential development of miRNAtargeting strategy as diagnostic and therapeutic tools for T2DM. The next review by Gwak and Park (2013) offers advanced knowledge of T2DM genetics based on recent GWA studies (Gwak and Park 2013). In particular, the authors highlight the genetic variants, which show significant associations with T2DM and functional implications in T2DM pathogenesis. The review also renders the potential clinical applications of these T2DM variants for the prediction of this disease, novel therapeutics, and personalized medication of anti-diabetic drugs. In addition to T2DM genetics, the article by Pak and colleagues suggests environmental chemicals as a T2DM pathogenic factor (Park et al. 2013b). Recent epidemiologic studies show that serum concentrations of environmental pollutants positively correlate with the incidence of age-related metabolic disorders, such as T2DM. They elaborate on the roles of synthetic pollutants, particularly focusing on aryl hydrocarbon receptor ligands, in the disturbance of mitochondrial function, which may be a major causative event of insulin resistance and T2DM. This novel concept of chemical-induced mitochondrial damage and its association with T2DM pathogenesis may offer a new way to develop therapeutic interventions. Lastly, Koo and colleagues have provided knowledge about the regulation of hepatic gluconeogenic program, focusing on transcriptional control (Oh et al. 2013). In this review, the authors introduce hepatic enzymes involved in glycogenolysis and gluconeogenesis, and their transcriptional regulations via transcription activators, co-activators, and repressors. In particular, there is an emphasis that $\mathrm{CREB}, \mathrm{CBP} / \mathrm{p} 300$, and CRTC2-dependent signaling induce the early phase gluconeogenic program, whereas transcriptional co-activators, which can be produced in the early phase gluconeogenesis, can in turn participate in the later phase gluconeogensis. Such provided insight into the regulation of hepatic glucose production can offer potential therapeutic targets for the treatment of T2DM.

The next four reviews are dedicated to illustrating the linkage between obesity and T2DM. As the first review, Jung and Kim (2013) illustrate the molecular mechanism of central leptin resistance in an attempt to understand obesity pathogenesis (Jung and Kim 2013). The adipocyte-derived hormone leptin primarily acts on the hypothalamus to activate catabolic pathways and to inhibit anabolic pathways, which in turn, results in appetite repression and weight reduction. Rather than leptin deficiency, hypothalamic leptin resistance has been suggested as a major contributing factor to obesity; therefore, molecular mechanisms of leptin resistance of hypothalamic neurons need to be uncovered. This review brings in the overview of leptin function and leptin receptor signaling, and fleshes out underlying molecular mechanisms of leptin resistance in hypothalamic neurons. Proposed mechanisms are: i) impaired leptin transport to the brain, ii) disrupted leptin receptor signaling in hypothalamic neurons, including SOCS3 and protein tyrosine phosphatase $1 \mathrm{~B}$, iii) sustained inflammation in hypothalamic neurons, and iv) enhanced ER stress and defective autophagy signaling in hypothalamic neurons. These updates will allow readers to understand the molecular mechanism of central leptin resistance, which can be a major cause for the development of T2DM. The second article by Lee (2013) finds the connecting factor between obesity and T2DM in chronic inflammation (Lee 2013). Chronic inflammation has received attention for playing an important role in the development of insulin resistance and T2DM. This relationship has been proved by the hypoglycemic effect of anti-inflammatory intervention in T2DM patients. This review extensively illustrates the role of inflammation in T2DM, and suggests that adipocyte tissue macrophages (ATM) can be a critical component in obesity-induced insulin resistance and T2DM by mediating inflammation. The author elegantly points up the role of macrophage in the immune system and its regulation, and discusses the function of ATM in obesity-induced inflammation and insulin resistance by delineating current knowledge in this field. Another explanation of the involvement of obesity in T2DM can be found in a review by Lee (2013) and colleagues, which highlights the role of autophagy in pancreatic $\beta$-cell functions (Quan et al. 2013). Autophagy is a process removing damaged/degenerated proteins or organelles within the cell and plays a crucial role in cellular homeostasis during energy starvation or environmental stress. From an angle that pancreatic $\beta$-cells are in high burden of insulin synthesis/secretion causing severe ER stress; autophagy has drawn great attention as a protective mechanism of $\beta$-cells. This review summarizes authors' recent findings, which support the role of autophagy in the progression of obesity to T2DM. The genetic mouse model with both autophagy deficiency and obesity develops severe T2DM, while autophagy-null mice do not develop diabetes. Additionally, authors discuss the role of hypothalamic neuronal autophagy in energy balance and appetite regulation, suggesting the importance of central autophagy regulation in obesity and diabetes. The fourth 
article by Koya and colleagues has underlined the role of Sirt1, an $\mathrm{NAD}^{+}$-dependent deacetylase, in diabetic nephropathy, which is the leading cause of end-stage kidney disease (Kume et al. 2013). Sirt1 has been recognized as an intracellular energy sensor and its dysregulation is associated with obesity, diabetes, and aging. Based on this, several Sirt1 activators have attracted attention as a novel anti-diabetic therapy. This review describes the role of Sirt1 in various kidney diseases, including aging-related lesions, renal hypoxic injury, renal interstitial fibrosis, along with renal inflammation, and proposes a therapeutic potential of Sirt1 activators for the treatment of diabetic nephropathy.

It is hoped that this special issue will stimulate scientists to continue to search for the factors and the mechanisms of diabetes and its complications, a devastating metabolic disease. Hopefully, these new ideas will translate into therapeutics, contributing to improvement in the prognosis of diabetic patients in near future. We are grateful to Drs. Sang Geon Kim and Aree Moon, Editors-in-Chief of Archives of Pharmacal Research, for giving us the opportunity to bring together these contributions in one issue. We also thank all the authors and their collaborators who contributed to this special issue.

\section{References}

http://www.diabetes.or.kr/temp/Diabetes_Fact_sheet2012.pdf. http://www.cdc.gov/diabetes/pubs/pdf/ndfs_2011.pdf.

Cheon, H.G. 2013. Latest research and development trends in noninsulin anti-diabetics. Arch. Pharm. Res 36: 2. doi:10.1007/ s12272-013-0016-7.

Gwak, S.H., and K.S. Park. 2013. Genetics of diabetes and its potential clinical application. Arch Pharm Res 36: 2. doi:10.1007/s12272013-0021-x.

Jung, C.H., and M.-S. Kim. 2013. Molecular mechanisms of central leptin resistance in obesity. Arch. Pharm. Res 36: 2. doi: 10.1007/s12272-013-0020-y.

Kume, S., M. Kitada, K. Kanasaki, H. Maegawa, and D. Koya. 2013. Anti-aging molecule, Sirt-1- A novel target for diabetic nephropathy. Arch. Pharm. Res 36: 2. doi:10.1007/s12272013-0019-4.

Lee, J. 2013. Adipose tissue macrophage in the development of obesity-induced inflammation, insulin resistance and type 2 diabetes. Arch. Pharm. Res 36: 2. doi:10.1007/s12272-0130023-8.

Oh, K.-J., H.-S. Han, M.-J. Kim, and S.-H. Koo. 2013. Transcriptional regulation of hepatic gluconeogenesis. Arch. Pharm. Res 36: 2. doi:10.1007/s12272-013-0018-5.

Park, S.-Y., H.-J. Jeong, W.-M. Yang, and W. Lee. 2013a. Implications of microRNAs in the pathogenesis of diabetes. Arch. Pharm. Res 36: 2. doi:10.1007/s12272-013-0017-6.

Park, W.-H., Y.-C. Kang, Y. Piao, D.H. Pak, and Y.K. Pak. 2013b. Causal effects of synthetic chemicals on mitochondrial deficits and diabetes pandemic. Arch. Pharm. Res 36: 2. doi:10.1007/ s12272-013-0022-9.

Quan, W., H.S. Jung, and M.-S. Lee. 2013. Role of autophagy in the progression from obesity to diabetes and in the control of energy balance. Arch. Pharm. Res 36: 2. doi:10.1007/s12272-0130024-7. 